\title{
Los sonidos fricativos sordos y sus implicaciones forenses
}

\author{
Voiceless fricative sounds and forensic implications
}

Jordi Cicres

Universitat de Girona, España. Correo electrónico: jordi.cicres@udg.edu

\begin{abstract}
Este artículo presenta los resultados de un estudio cuya finalidad es evaluar la capacidad de los sonidos fricativos sordos del español para discriminar entre hablantes en fonética forense. Se han analizado el centro de gravedad, la desviación estándar, la asimetría, la curtosis y el pico de mayor intensidad de los espectros de los segmentos fricativos, así como los valores estandarizados de las bandas LTAS. Los resultados permiten alcanzar de media el 89,78\% de casos originales agrupados correctamente, y el 71,6\% en la validación cruzada. Se discuten sus implicaciones teóricas, así como su aplicabilidad forense en identificaciones de hablantes con un conjunto cerrado de referencia.
\end{abstract} forense.

Palabras clave: identificación de hablantes, fricativas, análisis discriminante, fonética

This paper presents the results of a study aimed at assessing the capability of Spanish unvoiced fricative sounds to discriminate between speakers in forensic phonetics. The center of gravity, standard deviation, skewness, kurtosis and higher peak intensity of the spectra of fricative segments, as well as the standardized values of the bands LTAS have been analyzed. Results achieved on average $89.78 \%$ of original grouped cases correctly classified, and $71.6 \%$ in cross-validation. Theoretical implications are discussed, as well as its applicability in forensic speaker identification with a closed reference set.

Key words: speaker identification, fricatives, discriminant analysis, forensic phonetics.

\section{INTRODUCCIÓN}

Este estudio se circunscribe en el ámbito de la lingüística forense. Se trata de una disciplina relativamente moderna que estudia la interfaz entre el lenguaje y el derecho. En Gibbons y Turell (2008) se distinguen los tres grandes ámbitos de estudio de la linguiística forense: el lenguaje jurídico (the language of the law), el lenguaje judicial (the language of the court) y el lenguaje evidencial o probatorio (language as evidence). Los lingüistas forenses estudian, por lo tanto, los documentos legales (leyes, normas, contratos, pólizas de seguros, etc.), el lenguaje utilizado por los distintos actores de los procesos judiciales (jueces, abogados, testigos, peritos, inculpados, policías, etc.), los procesos de traducción e interpretación en contextos de apoyo multilingües, así como el lenguaje utilizado en los interrogatorios policiales. 
Sin embargo, es en el ámbito del lenguaje evidencial en el que los lingüistas desempeñan un papel de actores reales en los procesos judiciales como expertos en lingüística. Se les requiere para aportar sus conocimientos en los distintos niveles lingüísticos (fonética, fonología, morfología, sintaxis, semántica, pragmática, discurso, etc.) para aportar a los jueces los conocimientos técnicos y científicos necesarios con el fin de poder decidir en un procedimiento concreto. Entre las actuaciones que desempeñan los lingüistas forenses se encuentran las siguientes: comparar voces con el fin de identificar hablantes, determinar o atribuir la autoría de textos escritos, elaborar perfiles lingüísticos a partir de muestras de voz o de escritura, detectar y analizar casos de posibles plagios, o dictaminar en casos de litigio de marcas registradas y patentes.

Para desempeñar estas tareas, los lingüistas forenses describen aquellos aspectos del lenguaje que hacen a cada hablante o escritor único, el 'estilo idiolectal', que en palabras de Turell (2010) "[...] would have to do primarily, no with what system of language/dialect an individual has, but with a) how this system, shared by lots of people, is used in a distinctive way by a particular individual; b) the speaker/writer's production, which appears to be 'individual' and 'unique' (Coulthard, 2004) and also c) Halliday's (1989) proposal of 'options' and 'selections' from these options". En el campo de la fonética forense, además, este análisis es complementado con el estudio de las propiedades acústicas de la voz, que por razones fisiológicas es también única para cada hablante.

Sin embargo, cualquier acto lingüístico implica cierto grado de variación (Weinreich, Labov y Herzog, 1968), tanto en lo referente al uso del lenguaje como a las propiedades acústicas de la voz. Aun así, gran parte de esta variación está estructurada (Labov, 1966, 1972, 1994, 2001). Los factores que explican la variación pueden ser externos (sociales, estilísticos, geográficos, históricos o individuales) e internos (lingüísticos: fonéticos, fonológicos, morfológicos, sintácticos, semánticos, pragmáticos, discursivos), y son independientes entre sí en el sentido que eliminar o cambiar un factor interno únicamente incide en otros factores internos, mientras que los externos se mantienen igual, o viceversa (Turell, 2003).

Aunque la lingüística forense necesita estudiar todos los factores de variación, el factor individual es el más importante en las comparaciones forenses de textos con el fin último de identificar hablantes o escritores. Sin embargo, éste es el factor menos conocido. Por un lado, desde la sociolingüística de la variación (en especial desde la óptica de la escuela laboviana), son pocos los estudios que se centran en la variación individual (son excepciones los estudios de Guy, 1980; Coulthard, 2004; Abercrombie, 1969; y Biber, 1988, 1995). En cambio, son muy numerosos los que se han centrado en el análisis de los factores sociales como el sexo, la edad, los niveles educativo y económico, etc. Por otro lado, desde la lingüística tradicional, los estudios descriptivos se centran precisamente en lo que no varía para explicar el funcionamiento de la lengua, obviando en gran medida la variación intra e inter hablantes.

El objeto de análisis de este estudio es la variación intra e inter hablante de los sonidos fricativos sordos del español. Desde una óptica acústica descriptiva, la literatura que ha tratado estos sonidos se inicia con los estudios descriptivos de autores anglosajones como Hughes y Halle (1956), Harris (1958), Strevens (1960), Heinz y Stevens (1961) o Ladefoged (1975). Los primeros estudios para estas consonantes del español son Borzone de Manrique y Massone (1979 y 1981), Borzone de Manrique 
(1980), y Quilis (1981 y 1993). Estudios más recientes son los de Barreiro (2000) y Martínez-Celdrán y Fernández (2007).

Acústicamente, los sonidos fricativos se han estudiado básicamente desde tres puntos de vista: la estructura frecuencial, la duración e intensidad, y las transiciones formánticas, aunque siempre buscando los elementos invariantes y obviando la variación intra e inter hablante.

Respecto de su estructura espectral, en primer lugar, se ha estudiado el rango frecuencial del ruido, aunque ya en Quilis (1981: 221) se señala ya que "el ruido de fricción no basta para la identificación de todas las fricativas" del español, aunque es útil para distinguir las fricativa alveolar [s], prepalatal [J] y velar [x] -según Harris (1958), en la primera el umbral inferior en que aparecía el ruido de fricción estaba alrededor de los $3500 \mathrm{~Hz}$, mientras que en la segunda aparecía alrededor de los 2000 $\mathrm{Hz}$, y éstas de [f, $\theta$ ] en las que se podía observar energía en el espectrograma a partir de los $1000 \mathrm{~Hz}-$. Sin embargo, Quilis sitúa el inicio de la fricción en la labiodental en $\operatorname{los} 2100 \mathrm{~Hz}$ y en una horquilla entre los 2400 y los 6400 para la interdental, diferencia explicada por el "aumento del volumen de la cavidad anterior de resonancia, motivado por la labialización” (Quilis, 1981: 229). En cambio, Barreiro (2000: 247-248) sitúa el inicio de la fricción a los $1000 \mathrm{~Hz}$ para la labiodental y los 1100 para la interdental. Respecto del límite superior, Quilis no aporta datos, mientras que Barreiro señala los $13400 \mathrm{~Hz}$ para la labiodental y los 13500 para la interdental. Barreiro (2000: 247-248) y Martínez-Celdrán y Fernández (2007: 107-108) también han analizado los picos de mayor intensidad de cada sonido.

Más recientemente, otros estudios han analizado los cuatro primeros momentos espectrales (es decir, la media o centro de gravedad, la desviación estándar, la asimetría y la curtosis). Entre los principales estudios que han analizado estas variables destacan Fu et al. (1999) y Jongman et al. (1998) para el inglés, y Fernández et al. (1998) para el español.

En segundo lugar, se han estudiado también las transiciones formánticas, variables fundamentales para la distinción entre las fricativas no sibilantes (Quilis, 1981; Martínez-Celdrán y Fernández, 2007: 108). Y finalmente, se han observado diferencias en la duración e intensidad de cada grupo de fricativas, encontrándose diferencias muy significativas en la intensidad (siendo mayor en las fricativas [s] y [S]), y una tendencia a una mayor duración a medida que los sonidos fricativos son más posteriores, aunque muy dependientes de la velocidad de elocución y el contexto.

\section{OBJetivos E HIPÓTESIS}

Este estudio se ciñe al análisis en el contexto forense de las propiedades acústicas espectrales de un único grupo de sonidos (fricativos sordos). Sus objetivos son los siguientes:

a. Analizar la variación inter e intra hablante en los sonidos fricativos sordos del español peninsular para valorar su idoneidad como variables útiles en la comparación forense de voces.

b. Aplicar el análisis discriminante con el fin de determinar el potencial discriminador entre hablantes de los sonidos fricativos sordos. 
Para alcanzar dichos objetivos, se han formulado y testeado las siguientes hipótesis:

a. La variación intra hablante será menor que la variación inter hablante en las variables que describen espectralmente los sonidos fricativos.

b. El análisis discriminante permitirá discriminar entre hablantes, y se logrará un alto porcentaje de clasificaciones correctas de nuevos casos.

\section{Metodología}

\subsection{DESCRIPCIÓN DEL CORPUS}

Para el presente estudio se ha utilizado un corpus formado por seis hablantes masculinos, hablantes de español. Los informantes mantenían una conversación semiespontánea con el entrevistador, en una habitación silenciosa sin ninguna otra precaución acústica. La conversación se grababa mediante el micrófono interno de una grabadora Marantz Professional Solid State Recorder PMD671. Con posterioridad, se han identificado y segmentado los sonidos fricativos sordos [ $\mathrm{f} \theta \mathrm{s} \mathrm{x}$ ] en posición intervocálica. En total, se han utilizado para el estudio 658 segmentos distribuidos de forma no uniforme (según la disponibilidad de los contextos de análisis) entre los seis hablantes. La Tabla 1 resume la composición del corpus:

Tabla 1

Resumen del número de segmentos analizados según el hablante y la fricativa

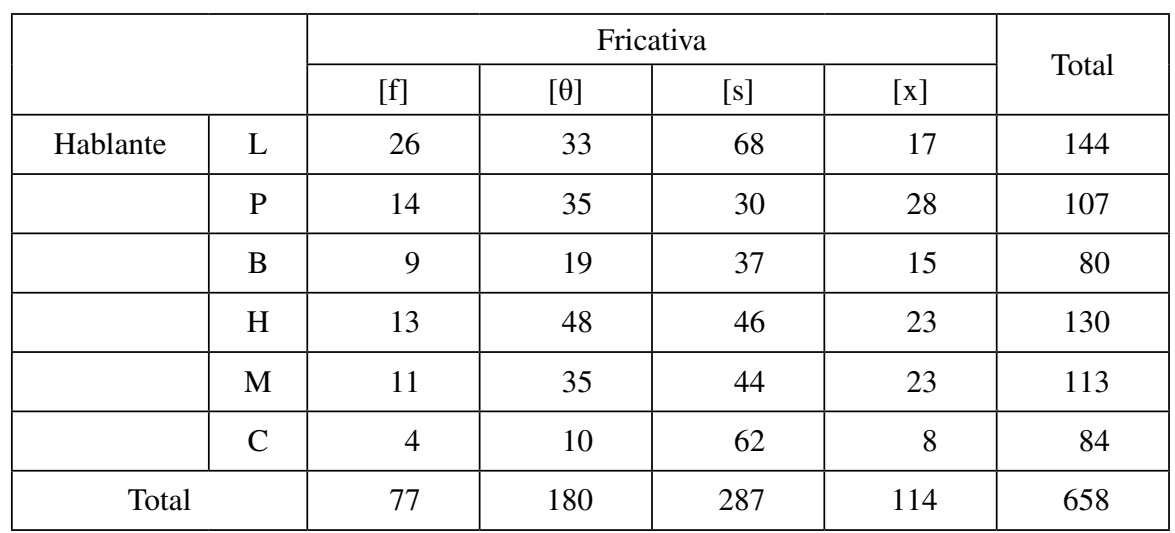

La segmentación se ha realizado manualmente mediante un textgrid del programa Praat (versión 5.2.42). Cada segmento es extraído de su contexto y guardado como un archivo independiente. En todos los casos, se sigue el siguiente protocolo:

i. Se reduce la frecuencia de muestreo a $32000 \mathrm{~Hz}$ (es la parte del espectro con información acústica para los sonidos fricativos). 
ii. Se aplica un filtro (Hass pass band) por el que se eliminan las frecuencias inferiores a los $500 \mathrm{~Hz}$. El objetivo de este filtrado es eliminar los ruidos de la grabación, así como los ruidos de fondo de baja frecuencia. En la práctica forense, habitualmente las grabaciones dubitadas son de baja calidad, con lo que el filtrado es imprescindible para la medición de parámetros espectrales globales.

A continuación, se ha programado un script por el que se extrae la información acústica del $50 \%$ central del segmento fricativo. De este modo, se reducen los efectos causados por la coarticulación y se eliminan del análisis fragmentos no fricativos, fruto de las posibles imprecisiones de la segmentación.

Los datos acústicos obtenidos por el script son los cuatro primeros momentos espectrales (media, desviación estándar, asimetría y curtosis), el punto de máxima intensidad en el espectro FFT y los valores de las bandas LTAS (de 1kHz) normalizadas para todo el espectro.

\subsection{VARIABLES DE ANÁLISIS}

Todas las variables de análisis utilizadas en este estudio hacen referencia a la forma del espectro. Concretamente, se analizan los cuatro momentos espectrales (media, desviación estándar, asimetría y curtosis), utilizados en estudios como Jongman et al. (1998) o Fu et al. (1999). También se utilizaron en Cicres (2004) en el contexto forense. Además, su independencia del contexto hace posible automatizar su análisis. Sin embargo, no son eficientes para discriminar entre [f] y [ $\theta]$ (Fu et al., 1999). Adicionalmente, se han utilizado como variables complementarias el punto de máxima intensidad en el espectro FFT y los valores de las 16 bandas LTAS (de 1kHz) a lo largo de todo el espectro. También se ha considerado como variable complementaria el valor máximo LTAS. A continuación se describen las distintas variables de análisis:

En primer lugar, el centro de gravedad (CG) de un espectro mide su concentración media de energía. Así, por ejemplo, para un espectro formado por una única onda simple de $1000 \mathrm{~Hz}$, su centro de gravedad es $1000 \mathrm{~Hz}$ (Figura 1); para un espectro formado por dos ondas simples de la misma intensidad a frecuencias de $1000 \mathrm{~Hz} \mathrm{y}$ $3000 \mathrm{~Hz}$, su centro de gravedad es $2000 \mathrm{~Hz}$ (Figura 2); o para un sonido blanco con una frecuencia de muestreo de $22050 \mathrm{~Hz}$, su centro de gravedad se sitúa en la mitad de la frecuencia Nyquist, es decir a $5512.5 \mathrm{~Hz}$.

La desviación estándar (el segundo momento espectral) mide la distancia de las frecuencias del espectro respecto del centro de gravedad. Para una onda simple, su desviación estándar es 0. Para un espectro con forma de M (como el de la Figura 3), la desviación estándar es elevada, ya que el centro de gravedad de encuentra en un punto intermedio a las dos zonas frecuenciales que concentran la mayor parte de la energía. En cambio, la desviación estándar del espectro de la Figura 4 es menor, puesto que el único pico de energía se corresponde con la zona del centro de gravedad.

Por su lado, la curtosis (tercer momento espectral) indica el grado de apuntamiento de la forma del espectro. Así, una curtosis positiva refleja espectros puntiagudos (Figura 5): "positive kurtosis thus suggests a clearly defined spectrum with wellresolved peaks, while negative kurtosis indicates a flat spectrum without clearly defined peaks" (Jongman et al., 2000: 1253). Valores negativos indican distribuciones más bien planas del espectro (Figura 6). 
Figura 1

Espectro de una onda simple con un centro de gravedad de $1000 \mathrm{~Hz}$

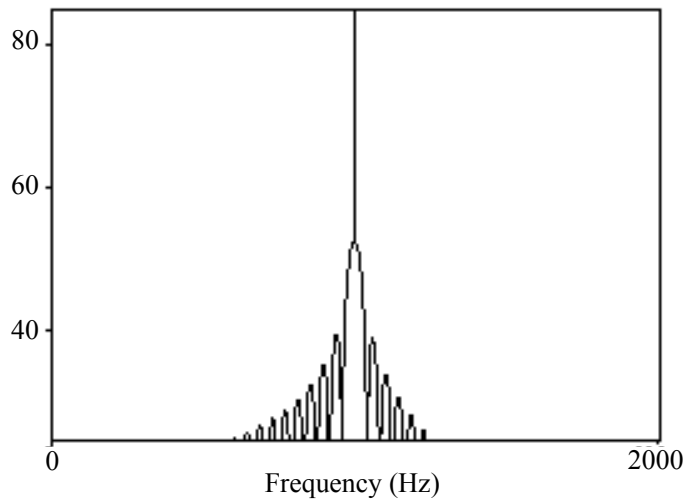

Figura 2

Espectro formado por dos ondas simples (de 1000 y $3000 \mathrm{~Hz}$ ) de la misma intensidad, con centro de gravedad de $2000 \mathrm{~Hz}$

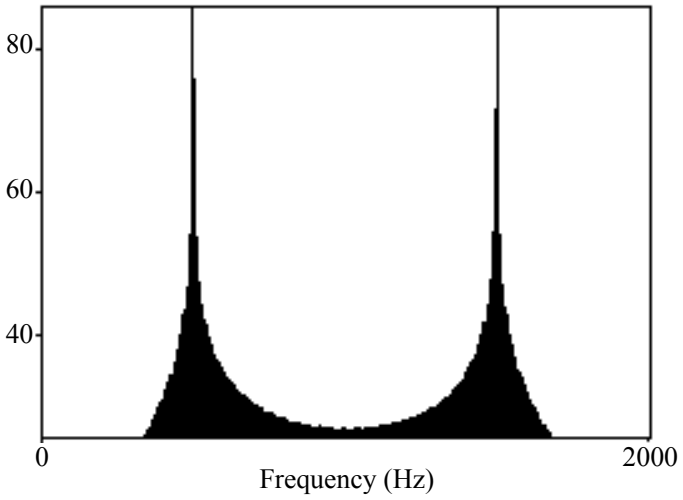

Figura 3

Espectro de una fricativa con centro de gravedad de $8291,85 \mathrm{~Hz}$ y desviación estándar de $4994,01 \mathrm{~Hz}$

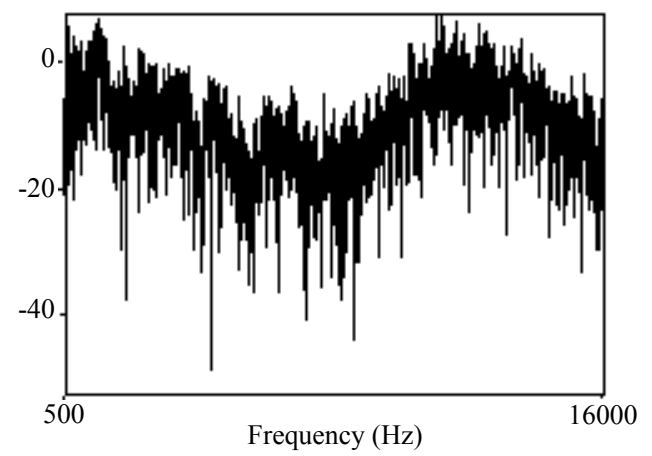


Figura 4

Espectro de una fricativa con centro de gravedad de $4857,15 \mathrm{~Hz}$ y desviación estándar de $1337,24 \mathrm{~Hz}$

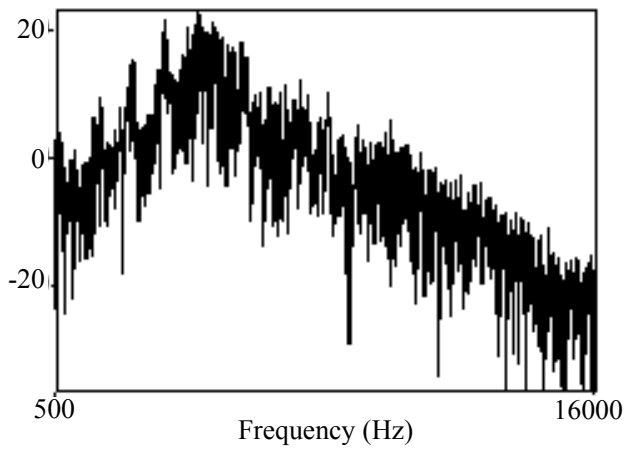

Figura 5

Espectro de una fricativa con curtosis positiva $(14,62)$

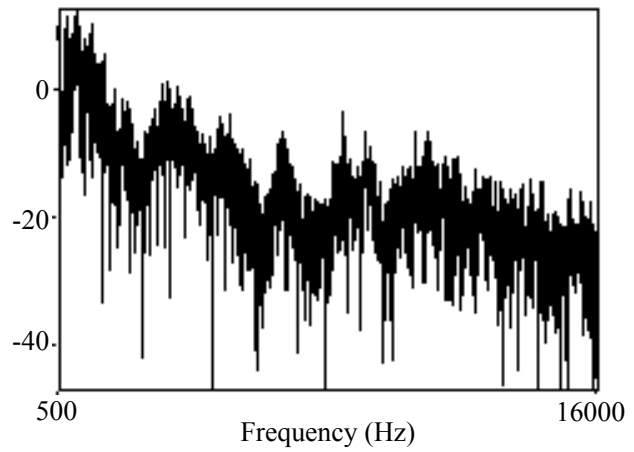

Figura 6

Espectro de una fricativa con curtosis negativa $(-1,21)$

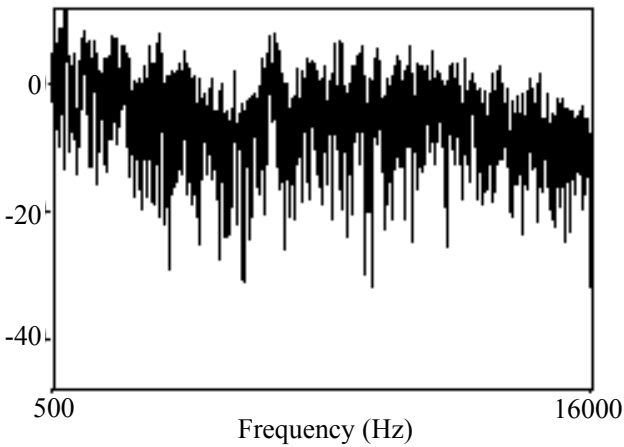


El cuarto momento espectral, la asimetría, está relacionado con la distribución de la energía a lado y lado del centro de gravedad, de modo que un espectro con asimetría 0 indica que la energía se distribuye de forma simétrica a ambos costados del centro de gravedad, mientras que si los valores son positivos el pendiente derecho de la distribución es mayor que el izquierdo. Es decir, los valores positivos indican mayor predominio de la energía en la zona de frecuencias bajas, mientras que los valores negativos aparecen en espectros con mayor energía en su parte alta. Las Figuras 7 y 8 muestran espectros de sonidos fricativos con valores de asimetría negativo y positivo respectivamente.

Adicionalmente, se ha obtenido el valor del Long-Term Average Spectrum (LTAS) mediante bandas de $1000 \mathrm{~Hz}$. El LTAS representa "[t]he average power in a sound during a certain time range and in a certain frequency range, expressed in $\mathrm{Pa}^{2} / \mathrm{Hz}$ " (Boersma y Weenick, 2011). Para evitar diferencias en estos valores que pudieran ser atribuidas a variables externas (proximidad al micrófono, diferencias en el ruido de fondo, volumen medio del hablante), los datos obtenidos del análisis LTAS se

Figura 7

Espectro de una fricativa con centro de gravedad de $5274,41 \mathrm{~Hz}$ y asimetría de $-1,32$

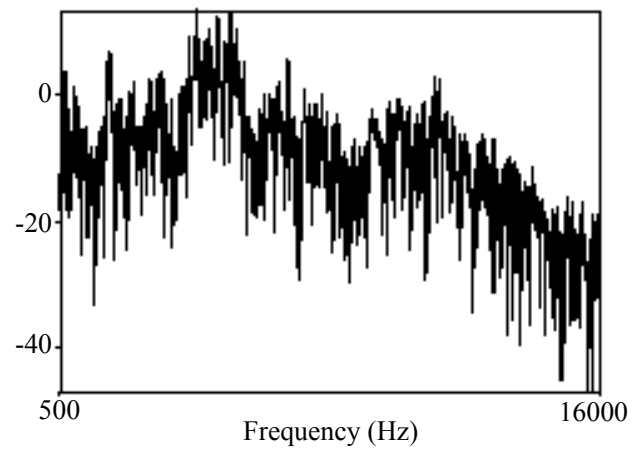

Figura 8

Espectro de una fricativa con centro de gravedad de $1518,32 \mathrm{~Hz}$ y asimetría de 4,1.

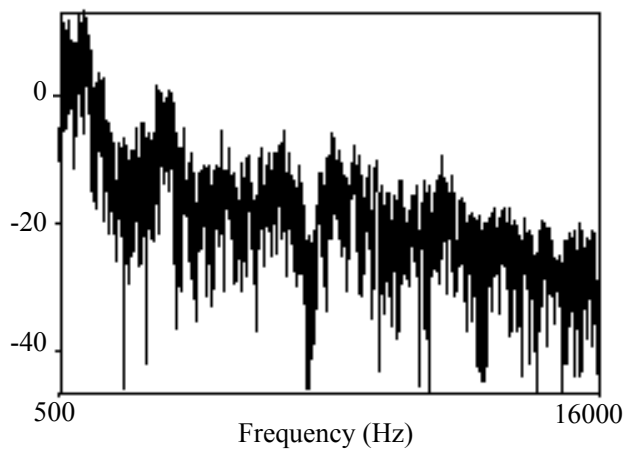


han estandarizado (de forma que para cada hablante los datos medios de cada banda tienen media 0 y desviación estándar 1). Finalmente, también se ha localizado el pico de mayor energía en el espectro FFT.

\subsection{ANÁLISIS ESTADÍSTICOS}

Para lograr los objetivos del estudio se han analizado los datos mediante un doble procedimiento estadístico. Por un lado, se ha utilizado el análisis de la varianza (ANOVA) con el fin de calcular la ratio de variación intra e inter hablante (hipótesis $a$ ). Para cada fricativa independientemente, se han utilizado las variables acústicas como variables dependientes y el hablante como factor independiente. Por el otro lado, para testear la hipótesis $b$, se han creado perfiles acústicos específicos para cada hablante y para cada fricativa independientemente. Como paso previo a la clasificación de nuevos casos, es posible testear el modelo mediante la validación cruzada. Este procedimiento consiste en recalcular la función discriminante una vez apartados del análisis un porcentaje de casos, que son clasificados en función de los valores de las variables utilizadas en la función. Si la clasificación de los casos es exitosa, se concluye que la función discriminante es la adecuada. Este proceso se puede repetir varias veces apartando casos distintos y analizando nuevamente si son clasificados correctamente con la nueva función discriminante. Una vez que la función discriminante ha sido validada, se puede proceder a la clasificación de casos nuevos con garantías de éxito.

\section{Resultados}

En primer lugar se presenta un resumen descriptivo de los datos. Se puede observar a partir de las medias estandarizadas de las bandas LTAS la distinta distribución de la energía en el espectro, que para las fricativas no sibilantes sigue un patrón muy parecido (de aquí su dificultad para discriminarlas a partir únicamente del análisis de sus propiedades espectrales), con mayor energía en las bandas altas, mientras que la fricativa alveolar presenta su mayor concentración de energía en la parte media del espectro; en cambio, la fricativa [x] sólo presenta valores superiores a la media de fricativas en las tres primeras bandas espectrales (Figura 9).

Por otro lado, la Tabla 2 muestra el resumen de las medias de los cuatro primeros momentos espectrales y del valor del pico máximo del espectro FFT, y las Figuras 10 a 13 muestran los diagramas de caja de estas variables. Se puede observar sobre todo en las fricativas no sibilantes ([f] y [ $[\theta]$ ) que la variabilidad intra e inter hablante es alta, mientras que en [s] y [x] los valores son claramente más homogéneos.

Sin embargo, para los objetivos del presente estudio (hipótesis $a$ ), es necesario comparar estadísticamente la ratio de variación intra e inter hablante. El análisis ANOVA presenta diferencias estadísticamente significativas $(\mathrm{p}<0,05)$ entre hablantes para la mayoría de las variables del estudio (incluso para los valores estandarizados de las bandas LTAS). Únicamente no se encuentran diferencias inter hablantes significativas en la quinta banda del LTAS para [f]; las tres primeras bandas para [ $\theta$ ]; las bandas 8 a 10 de [s]; y el centro de gravedad, el valor máximo del espectro FFT, la banda LTAS de mayor intensidad y las bandas 1 a 4 y 13 a 16 en el caso de [x]. Mediante las pruebas 
Figura 9

Media de energía (valores estandarizados) para cada banda LTAS

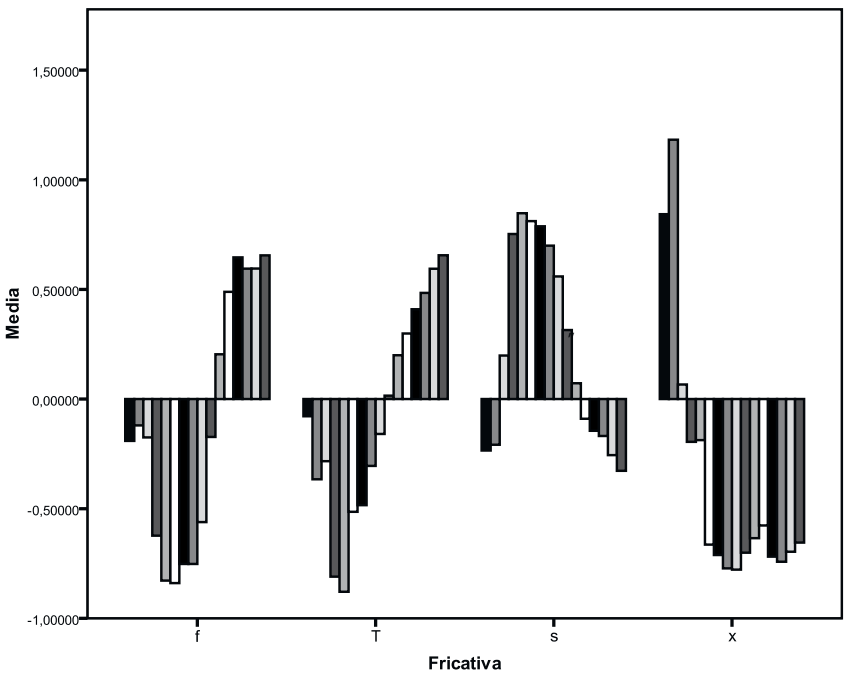

Zscore(ttas01)

Zscore(ltas02)

Zscore(tas 04

Zscore(ttas05)

Zscore(ttas06)

Zscore(tias07)
Zscore(tas08)

Zzscore(tas09)

Zzscore(tlas 10)

Zscore(ttas 12)

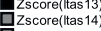

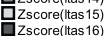

Tabla 2

Resumen de los datos medios, en Hz.

\begin{tabular}{|c|c|c|c|c|c|c|c|}
\hline & \multicolumn{6}{|c|}{ Hablante } \\
\hline & & $\mathrm{L}$ & $P$ & B & $\mathrm{H}$ & $M$ & $\mathrm{C}$ \\
\hline \multirow{5}{*}{ [f] } & Centro de gravedad & 7634,60 & 4540,69 & 8014,15 & 6341,42 & 7166,28 & 3492,29 \\
\hline & Desviación estándar & 3939,00 & 3562,98 & 4263,85 & 3877,13 & 4362,86 & 2643,39 \\
\hline & Curtosis &,- 55 & 2,02 &,- 82 &,- 60 &,- 87 & 7,28 \\
\hline & Asimetría &,- 35 & 1,27 &,- 21 & ,48 &, 10 & 2,06 \\
\hline & Valor máximo FFT & 7044,77 & 2521,21 & 7048,61 & 3990,38 & 5600,85 & 2398,44 \\
\hline \multirow{5}{*}[\theta]{} & Centro de gravedad & 7624,65 & 6023,04 & 5867,76 & 6125,78 & 6801,62 & 4542,93 \\
\hline & Desviación estándar & 3709,10 & 4050,24 & 4561,84 & 3635,08 & 3658,26 & 3121,78 \\
\hline & Curtosis &,- 56 &,- 38 &,- 38 &,- 06 &,- 71 & 3,97 \\
\hline & Asimetría &,- 31 & , 48 & 61 & 49 &, 23 & 1,44 \\
\hline & Valor máximo FFT & 5093,28 & 2938,39 & 1260,69 & 3933,92 & 4715,18 & 2907,81 \\
\hline \multirow{5}{*}[\mathrm{s}]{} & Centro de gravedad & 5911,75 & 5121,15 & 5102,65 & 4827,36 & 4894,00 & 5529,53 \\
\hline & Desviación estándar & 2236,18 & 2352,09 & 2024,56 & 1552,20 & 1645,39 & 1782,07 \\
\hline & Curtosis &, 41 & 1,15 & 4,49 & 6,75 & 5,97 & 4,59 \\
\hline & Asimetría &, 25 & 84 & 1,42 & 1,19 & 1,85 & ,92 \\
\hline & Valor máximo FFT & 4791,93 & 4130,73 & 3972,34 & 4439,37 & 4300,78 & 4901,08 \\
\hline \multirow[t]{5}{*}[\mathrm{x}]{} & Centro de gravedad & 3344,40 & 2722,58 & 2513,56 & 2974,70 & 2684,44 & 2738,63 \\
\hline & Desviación estándar & 2604,86 & 2401,36 & 2419,60 & 2274,59 & 2067,86 & 1849,10 \\
\hline & Curtosis & 3,12 & 7,35 & 6,90 & 6,53 & 12,83 & 13,64 \\
\hline & Asimetría & 1,70 & 2,44 & 2,49 & 2,23 & 2,96 & 3,08 \\
\hline & Valor máximo FFT & 1973,35 & 1517,30 & 1404,17 & 1775,81 & 1562,50 & 2255,86 \\
\hline
\end{tabular}


Figura 10

Diagramas de caja del centro de gravedad, por fricativa y hablante

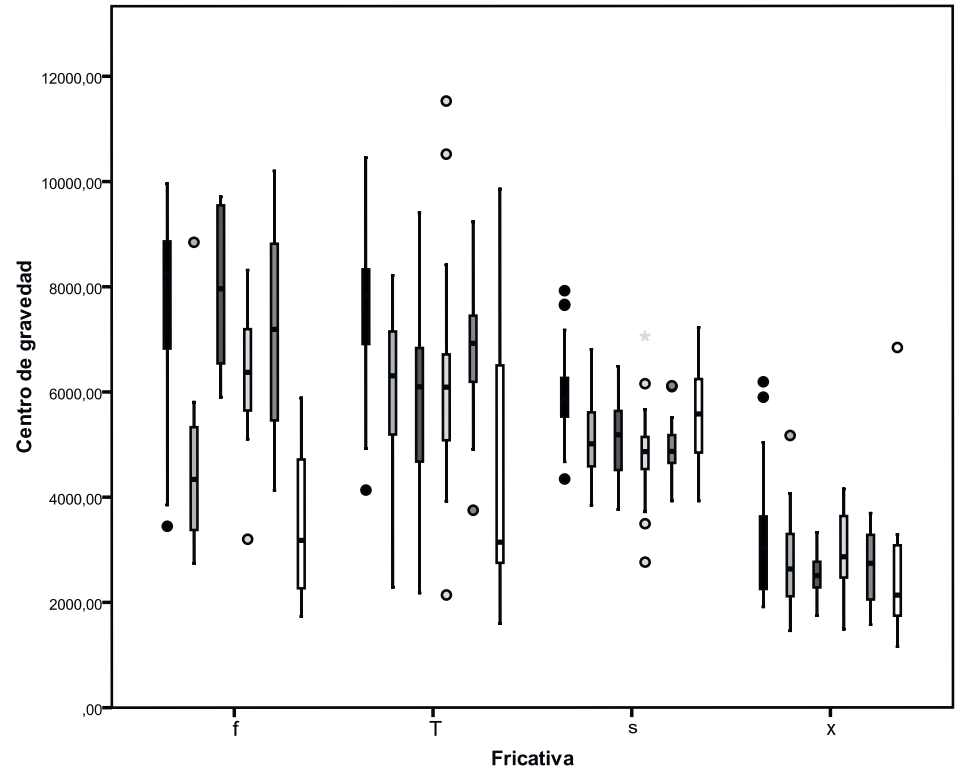

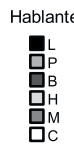

Figura 11

Diagramas de caja de la desviación estándar, por fricativa y hablante

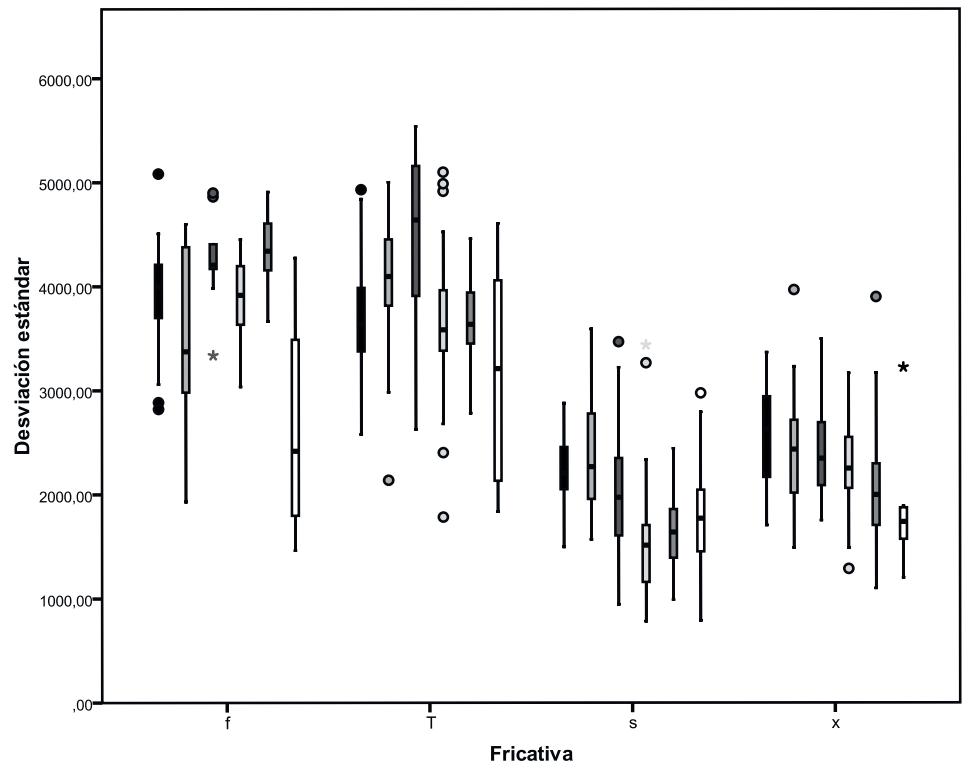

Hablant
昌
$\mathrm{Q}_{\mathrm{B}}$
$\mathrm{B}_{\mathrm{B}}^{\mathrm{H}}$
$\mathrm{G}_{\mathrm{M}}$ 
Figura 12

Diagramas de caja de la curtosis, por fricativa y hablante

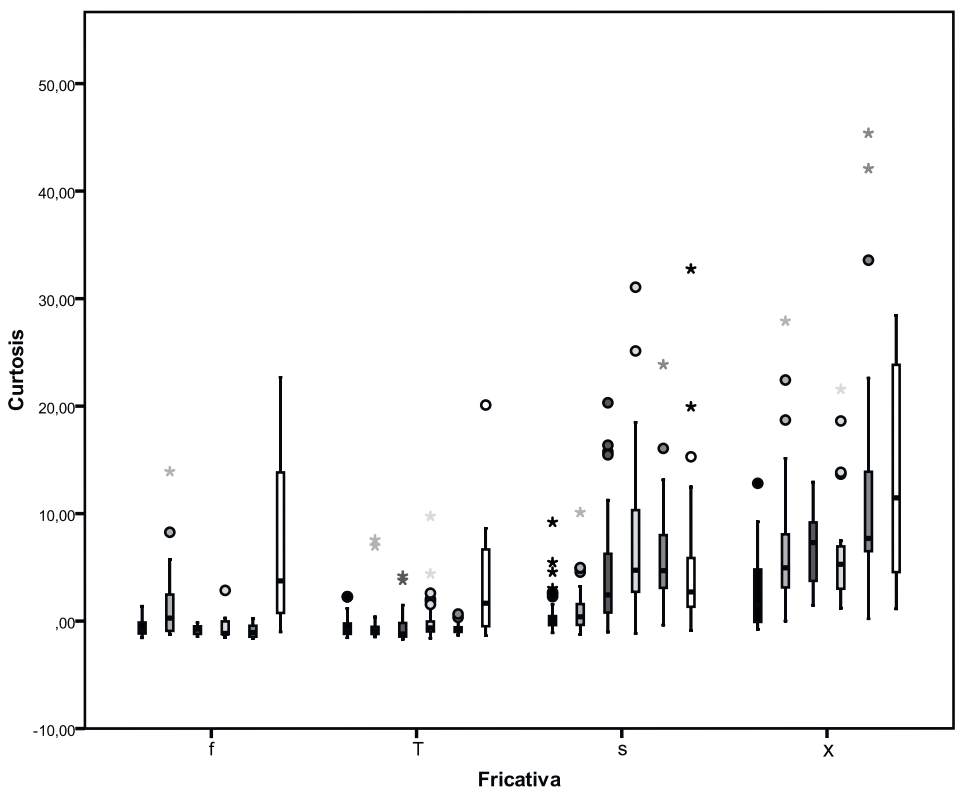

品
品
品
品

Figura 13

Diagramas de caja de la asimetría, por fricativa y hablante

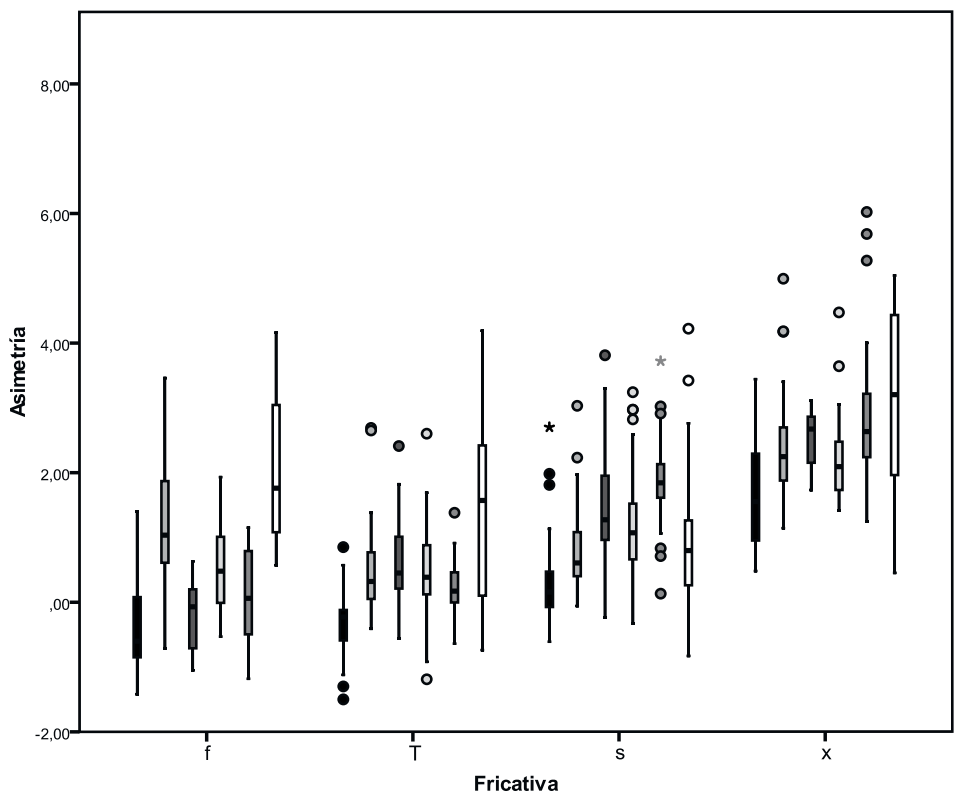

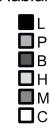


post-hoc (Tukey, cuando se asume la igualdad de varianzas entre hablantes, o GamesHowell en caso contrario) se observan diferencias estadísticamente significativas que permiten diferenciar entre todos los hablantes (con diferencias significativas en por lo menos alguna variable). Los resultados de las pruebas ANOVA permiten validar la hipótesis $a$ en el sentido que efectivamente la variación inter hablante es mayor que la variación intra hablante.

Finalmente, los datos del análisis discriminante (que permite testear la hipótesis $b$, según la cual el análisis discriminante debería discriminar entre hablantes con un alto porcentaje de clasificaciones correctas de nuevos casos) muestran un notable éxito.

La Figura 14 muetra el peso que tienen las variables del análisis en las cinco funciones discriminantes utilizadas en el análisis. En las dos primeras funciones (las que permiten explicar mayor variación) tienen un peso fundamental la asimetría y la desviación estándar (con valores positivos) y la curtosis (negativos); aunque con menor peso, también destacan las bandas LTAS 5 a 8 y 11, 12 y 14. En la segunda función discriminante el mayor peso lo tienen el centro de gravedad, la asimetría y las dos primeras bandas LTAS. Las demás funciones tienen un peso menor en la clasificación.

Mediante estas funciones, se logra una media del $89,78 \%$ de correspondencia entre los grupos pronosticados siguiendo el modelo discriminante y los grupos reales. Sin embargo, no es hasta que se clasifican nuevos casos que se puede evaluar realmente la capacidad discriminante de las funciones. Para ello, se pueden añadir nuevos casos conocidos, y comprobar si quedan bien clasificados según las funciones discriminantes, o bien se puede utilizar el método de clasificación dejando uno fuera (validación cruzada), que consiste en eliminar casos uno a uno del modelo y clasificarlos según las funciones discriminantes resultantes del resto de casos. Con este método se

Figura 14

Resumen de los valores de las funciones discriminantes para cada variable

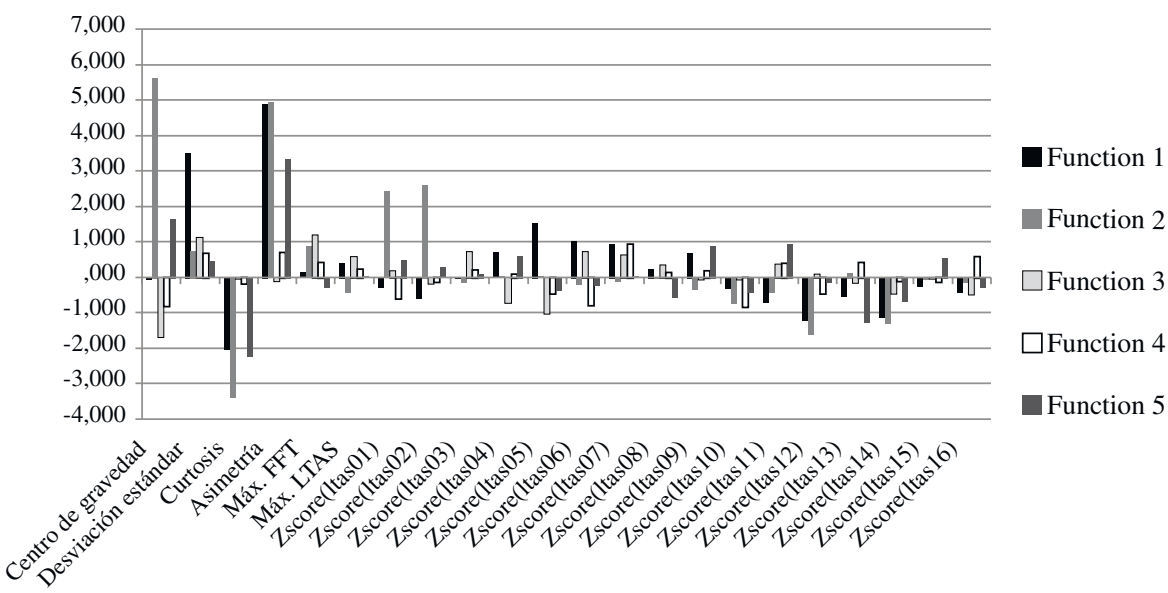


logra un 71,6\% de clasificaciones correctas (Tabla 3), porcentaje elevado dadas las probabilidades de clasificación al azar, que son de poco más del 16\%.

Tabla 3

Resumen de los resultados del análisis discriminante. Porcentaje de casos clasificados correctamente en el modelo y en la validación cruzada

\begin{tabular}{|c|c|c|}
\cline { 2 - 3 } \multicolumn{1}{c|}{} & $\begin{array}{c}\text { \% de casos clasificados } \\
\text { correctamente en el modelo }\end{array}$ & $\begin{array}{c}\text { \% de casos clasificados correctamente } \\
\text { en la validación cruzada }\end{array}$ \\
\hline$[\mathrm{s}]$ & 86,8 & 79,4 \\
\hline$[\mathrm{x}]$ & 91,2 & 71,9 \\
\hline$[\theta]$ & 88,9 & 76,7 \\
\hline$[\mathrm{f}]$ & 92,2 & 58,4 \\
\hline media & 89,78 & 71,6 \\
\hline
\end{tabular}

No obstante, hay que hacer algunas consideraciones acerca de los resultados de clasificación. Para [f], en tres de los hablantes el porcentaje de clasificaciones correctas (en clasificaciones de nuevos casos) era inferior al 50\%. Se trata del hablante C ( $25 \%$, y confundido con L, P y M), el hablante B (44\%, confundido mayoritariamente con M), y el hablante M (45\%, que fue confundido básicamente con L y B). Para la interdental, el hablante B fue clasificado correctamente en solo el $47 \%$ de las realizaciones (y los errores se dan básicamente con $\mathrm{H}$ ); el hablante $\mathrm{C}$ se clasificó correctamente en la mitad de los casos, y la mayoría de errores fueron con el hablante L. Los porcentajes de clasificación correctas para [s] se sitúan para todos los hablantes entre el 78 y el $91 \%$ excepto para el hablante B, que logra un $32 \%$ de casos clasificados correctamente (frente a un 35\% de casos erróneamente clasificados en M). Finalmente, el análisis de los resultados de clasificación de $[\mathrm{x}]$ muestran en todos los casos clasificaciones correctas superiores al $60 \%$.

\section{DisCUSIÓN Y CONCLUSIONES}

A la luz de los resultados obtenidos, se puede concluir que el análisis de la estructura espectral de los sonidos fricativos sordos del español es útil para el trabajo forense de comparación o identificación de voces.

Por un lado, se ha demostrado que la variación inter hablante es mayor que la variación intra hablante en prácticamente la totalidad de las variables analizadas, lo que apoya el carácter individual y único de la voz.

Por otro lado, el potencial discriminatorio de las variables analizadas referentes a la estructura espectral de los sonidos fricativos se ha mostrado elevado (con un $71,6 \%$ de clasificaciones correctas de nuevos casos). Sin embargo, la aplicación de la metodología utilizada es idónea únicamente para tareas de identificación de hablantes con corpus de referencia cerrado (Rose, 2002), aunque con un corpus de referencia suficientemente representativo de la población comparable a la voz dubitada las 
variables de análisis podrían ser de interés en el cálculo de las ratios de verisimilitud en el entorno bayesiano. También hay que tener en cuenta que la mayor parte del rango frecuencial analizado desaparece por el efecto del filtro telefónico (que filtra las frecuencias inferiores a $300 \mathrm{~Hz}$ y superiores a $3400 \mathrm{~Hz}$ ), y que las variables son sensibles a posibles diferencias en el nivel de ruido de fondo, o a métodos de compresión de audio (con pérdidas).

Finalmente, cabe destacar que, con el actual estado del arte en comparación forense de voces o de textos escritos, los peritajes lingüísticos comprenden una batería -cualitativa y cuantitativa- de mediciones y análisis, por lo que en ningún caso el análisis de un único grupo de variables (como en este ejemplo, la estructura espectral de los sonidos fricativos sordos), o únicamente una valoración cuantitativa o estadística puede ser suficiente para elaborar un dictamen pericial.

\section{OBRAS CITADAS}

Abercrombie, D. 1969. "Voice qualities". En Markel, N.N. (ed.) Psycholinguistics: an Introduction to the Study of Speech and Personality. London: The Dorsey Press.

Barreiro, S. C. 1999-2000. "Análisis acústico comparado de las fricativas castellanas no sibilantes en realizaciones aisladas". Contextos 33-36: 243-261.

Biber, D. 1988. Variation across Speech and Writing. Cambridge: CUP.

-. 1995. Dimensions of Register Variation: a Cross-linguistic Comparison. Cambridge: CUP.

Boersma, P. y D. Weenink. 2011. Praat: doing phonetics by computer [Programa informático]. Versión 5.2.44. http://www.praat.org/.

Borzone de Manrique, A.M. y M.I. Massone. 1981. "Acoustic analysis and perception of the Spanish fricative consonants". Journal of the Acoustical Society of America 69: 11451153.

Borzone de Manrique, A.M. 1980. Manual de fonética acústica. Buenos Aires: Hachette.

Coulthard, M. 2004. "Author Identification, Idiolect, and Linguistic Uniqueness". Applied Linguistics 25 (4): 431-447.

Fu, H., R. Rodman, D. McAllister, D. Bitzer y B. Xu. 1999. "Classification of Voiceless Fricatives through Spectral Moments". Proceedings of the 5th International Conference on Information Systems Analysis and Synthesis (ISAS'99). Skokie, Ill.: International Institute of Informatics and Systemics. 307-311.

Gibbons, J. y M.T. Turell (eds.). 2008. Dimensions of Forensic Linguistics. Amsterdam/ Philadelphia: John Benjamins.

Guy, G. 1980. "Variation in the group and the individual". En Labov, W. (ed.). Locating language in time and space. New York: Academic Press. 1-36.

Halliday, M.A.K. 1989. Spoken and Written Language. Oxford: Oxford University Press.

Harris, K.S. 1958. "Cues for the Discrimination of American English Fricatives in Spoken Syllables". Language and Speech 1: 1-7.

Heinz, J.M. y K.N. Stevens. 1961. "On the Properties of Voiceless Fricatives Consonants". Journal of the Acoustical Society of America 33: 589-96.

Hughes, G.W., M. Halle. "Spectral properties of fricative consonants". Journal of the Acoustical Society of America 28: 303-310.

Jongman, A., R. Wayland y S. Wong. 2000. "Acoustic characteristics of English fricatives". Journal of Acoustic Society of America 108 (3): 1252-1263. 
Labov, W. 1966. The social stratification of English in New York City. Washington D.C.: Center for Applied Linguistics.

—. 1972. Sociolinguistic Patters. Philadelphia: Pennsylvania University Press.

- 1994. Principles of Linguistic Change, I: Internal Factors. Oxford: Blackwell.

-. 2001. Principles of Linguistic Change, II: External Factors. Oxford: Blackwell.

Ladefoged, P. 1975. A course in phonetics. Orlando: Harcourt Brace.

Martínez Celdrán, E. y A. M. Fernández Planas. 2007. Manual de fonética española. Articulaciones y sonidos del español. Barcelona: Ariel.

Quilis, A. 1981. Fonética acústica de la lengua española. Madrid: Gredos.

—. 1993. Tratado de fonología y fonética españolas. Madrid: Gredos.

Rose, Ph. 2002. Forensic Speaker Identification. London: Taylor \& Francis.

Strevens, P. 1960. "Spectra of fricative noise in human speech". Language and Speech 3: 32-49.

Turell, M.T. 2003. "El temps aparent i el temps real en estudis de variació i canvi lingüístic". Noves SL. Revista de Sociolingüística. $<$ http://www6.gencat.net/llengcat/noves/hm03tardor/turell4_4.htm >

-. 2010. 'The use of textual, grammatical and sociolinguistic evidence in forensic text comparison". The International Journal of Speech, Language and the Law 17 (2): 211-250.

Weinreich, U., W. Labov y M. Herzog. 1968. "Empirical foundations for a theory of language change". En Lehmann, W. e Y. Malkiel (ed.). Directions for Historical Linguistics. Austin: University of Texas Press. 